\title{
SUBCARRIER POWER ADJUSTMENT TECHNIQUE FOR PEAK-TO-AVERAGE POWER RATIO REDUCTION OF OFDM SYSTEMS
}

\author{
Rakesh Rajbanshi, Alexander M. Wyglinski and Gary J. Minden \\ Information and Telecommunication Technology Center \\ The University of Kansas, Lawrence, KS 66045 \\ Email: $\{r a j b a n s h$, alexw, gminden\}@ittc.ku.edu
}

\begin{abstract}
In this paper, we propose a novel peakto-average power ratio (PAPR) reduction algorithm for OFDM systems that employs a two-step approach for adjusting the subcarrier power levels. The first step redistributes the power level across all subcarriers, which slightly reduces the error robustness of the system, while the second step ensures the subcarrier power distribution obeys a subband power constraint. The resulting power levels satisfy spectrum regulatory requirements across the transmission spectrum while simultaneously reducing the PAPR and maintaining a degree of error robustness. Since no overhead information is required, the proposed algorithm does not incur a throughput penalty. Simulation results for a 256-subcarrier OFDM system employing QPSK symbols and the proposed algorithm show a PAPR reduction of $1.5 \mathrm{~dB}$ for a complementary cumulative distribution of $0.1 \%$.
\end{abstract}

\section{INTRODUCTION}

Multicarrier modulation, such as orthogonal frequency division multiplexing (OFDM), is widely deployed in high speed data transceivers, for e.g. xDSL [1], IEEE 802.11a [2], IEEE 802.11g [3], IEEE 802.16a [4], due to its ability to efficiently handle the distortions introduced by frequency selective fading channels [5]. Due to the summation of OFDM subcarriers at the transmitter, the time domain OFDM symbol could exhibit large envelope variations when input sequence is highly correlated [6, 7]. This is often characterized by a large peak-to-average power ratio (PAPR) [8]. When high PAPR occurs, the digital-to-analog (D/A) converter and power amplifier of the transmitter would require a large dynamic range to avoid amplitude clipping, thus increasing both power consumption and component cost of the transceiver.

This work was supported by NSF grants ANI-0230786 and ANI-0335272.
Several subcarrier power adjustment techniques for reducing PAPR have been proposed in the literature, including iterative input sequence envelope scaling [9], weighted OFDM [10], weighted OFDM with block coding [11], frequency domain weighting [12], and active constellation extension [13]. Iterative techniques, such as input sequence envelope scaling, exhibit slow convergence times for finding optimal power scaling factors to minimize PAPR. Furthermore, computationally expensive FFT operations are repeated. The weighted OFDM approach reduces PAPR at the cost of serious bit error performance degradation, whereas the weighted OFDM with block coding technique is only suitable for systems with a small number of subcarriers since the algorithm must search for an optimal code with minimum PAPR over all possible codewords. Similarly, active constellation extension techniques achieve high reduction in PAPR, however, these algorithms may violate practical transmit power constraints.

In this paper, we propose a novel subcarrier power adjusting PAPR reduction algorithm for MPSK-OFDM, in which subcarrier power levels are altered to minimize PAPR. Information regarding the power level adjustments do not need to be transmitted to the receiver. Therefore, there is no throughput penalty. Moreover, the power adjustments obey a subband power constraint. Therefore, the system would not violate regulatory power requirements. However, the PAPR reduction comes at the cost of slight BER performance degradation.

The rest of the paper is organized as follows: In Section II, we present system framework for OFDM system. In Section III, we present definition of PAPR. In Section IV, we analyze subcarrier power adjusting PAPR reduction techniques. In Section V, the proposed subcarrier power adjustment algorithm for reducing PAPR of an OFDM signal is presented. In Section VI, several 


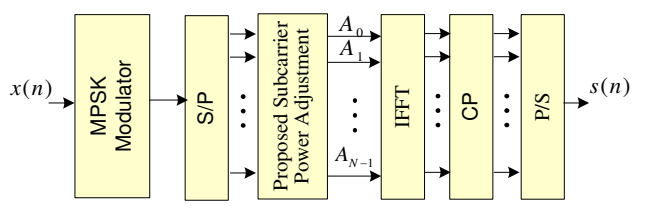

(a) OFDM Transmitter.

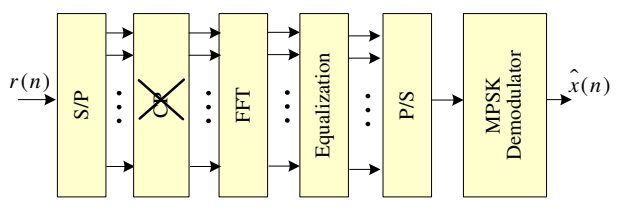

(b) OFDM Receiver.

Fig. 1. OFDM Transceiver employing subcarrier power adjustment for PAPR reduction.

simulation results are discussed and several conclusions are drawn in Section VII.

\section{SYSTEM FRAMEWORK}

A schematic of an OFDM transceiver employing the proposed PAPR reduction algorithm is shown in Fig. 1. The basic principle of OFDM is to split a high-speed data stream, $x(n)$, into slower data streams that are transmitted simultaneously over $N$ orthogonal subcarriers. Input data stream are firstly modulated using phase shift keying $(\mathrm{PSK})^{1}$. The proposed subcarrier power adjustment algorithm assigns the subcarrier power levels to minimize the PAPR of an OFDM signal. The inverse fast Fourier transform (IFFT) is then used to transform these modulated subcarrier signals into the time domain. Prior to transmission, a guard interval, with a length greater than the channel delay spread, is added to each OFDM symbol using the cyclic prefix (CP) block in order to mitigate the effects of intersymbol interference (ISI). Following the parallel-to-serial (P/S) conversion, the baseband OFDM signal, $s(n)$, is then passed through the transmitter radio frequency (RF) chain, which amplifies the signal and upconverts it to the desired center frequency.

The receiver performs reverse operation of the transmitter, mixing the RF signal to baseband for processing, yielding the signal $r(n)$. Then, the signal is converted into parallel streams, the cyclic prefix is discarded, and the fast Fourier transform (FFT) is applied to transform the time domain data into the frequency domain. After the distortion from the channel has been compensated per subcarrier via equalization. The parallel stream is

\footnotetext{
${ }^{1}$ In quadrature amplitude modulation, power adjustment information needs to be transmitted to the receiver for correct demodulation.
}

converted into serial stream using $\mathrm{P} / \mathrm{S}$ converter. The subcarrier data is demodulated and converted into a reconstructed version of the original high-speed input, $\hat{x}(n)$.

\section{Peak-to-Average Power Ratio}

The complex envelope of a baseband OFDM signal, consisting of $N$ subcarriers over a time interval $[0, T]$, is given by:

$$
s(t)=\frac{1}{N} \sum_{k=0}^{N-1} A_{k} e^{j 2 \pi k t / T}
$$

where, $A_{k}$ is the symbol of the $k^{\text {th }}$ subcarrier $^{2}, T$ is the OFDM symbol duration, and $j=\sqrt{-1}$. Without loss of generality, we can safely neglect the cyclic extension from the analysis since it does not contribute to the PAPR problem.

The PAPR of Eq. (1) is defined as the ratio between the maximum instantaneous power and the average power, namely [8]:

$$
\operatorname{PAPR}(s)=\frac{\max _{0 \leq t \leq T}|s(t)|^{2}}{E\left\{|s(t)|^{2}\right\}}
$$

where $E\{$.$\} denotes the expectation operator. The$ continuous-time PAPR of $s(t)$ can be approximated using the discrete-time PAPR, which is obtained using samples of the OFDM signal ${ }^{3}$. In this paper, the discrete time PAPR will be employed in the analysis.

PAPR needs to be minimized to improve the efficiency and minimize cost of the power amplifiers and D/A converters. A PAPR reduction algorithm ideally should achieve high PAPR reduction rapidly with low computational complexity. Moreover, keeping side information to a minimum, even eliminating it, is desired to avoid any throughput loss, as well as keep transmit power levels to a minimum without any BER performance degradation. Finally, the algorithm should not cause any distortion of the signal. Various algorithms have been proposed in literature which achieve some of the above attributes at the expense of other attributes.

\section{Subcarrier Power Adjustment}

In a multicarrier transmission system, power loading is a powerful technique for enhancing system performance when the system operates in a frequency selective

\footnotetext{
${ }^{2}$ For example, $A_{k} \in\{0,1\}$ for BPSK signaling and $A_{k} \in$ $\{ \pm 1, \pm j\}$ for QPSK signaling.

${ }^{3}$ It has been shown that an oversampling factor of four is sufficient to estimate the continuous PAPR of a BPSK system $[14,15]$.
} 
fading channel. In power loading, the power distribution across all the subcarriers vary according to the estimated channel conditions in order to minimize the overall error probability [16]. It has been shown in the literature that PAPR can be minimized by adjusting subcarrier power levels [9-13]. In this paper, we focus on the following two power adjustment approaches that will be employed by the proposed PAPR reduction:

1) Total power constraint $[17,18]$

2) Window power constraint $[16,19]$

In the following two subsections, these approaches will be described in detail.

\section{A. Total Power Constraint}

Total power-constrained power adjustment implies that if power of any subcarrier is reduced or turned off, the excess power allocated to it can be transferred to remaining active subcarriers. Let $\pi_{k}$ be the transmitted power of the $k$-th subcarrier $(k=0,1, \ldots, N-1)$. If the total number of subcarriers is $N$, the power constraint is given by:

$$
\sum_{k=0}^{N-1} \pi_{k}=\pi_{\text {total }} .
$$

The aggregate bit rate is approximately maximized if the bit error rates in all the sub-bands are equal, whereas BER performance is optimized when all the subcarriers have equal power [20]. Without power adjustment, $\pi_{k}$ is assumed to be equal for all subcarriers. In case of total power-constrained power adjustment, it is possible that all the power could be concentrated to a single subcarrier. However, such an allocation could potentially violate the spectrum regulations requirements. The following power constraint is designed to avoid these violations.

\section{B. Window Power Constraint}

If the power level of certain subcarrier is lowered, in window power-constrained power adjustment the excess power can be transferred to the active subcarriers within certain predefined sliding window. The total power for every grouping of $M$ subcarriers needs to be below the regulatory requirement, say $\pi_{\max }$. Then, the subcarrier power constraints would be:

$$
\sum_{k=l}^{l+M-1} \pi_{k} \leq \pi_{\max }, \quad \forall l
$$

and

$$
\sum_{k=0}^{N-1} \pi_{k} \leq \pi_{\text {total }} .
$$

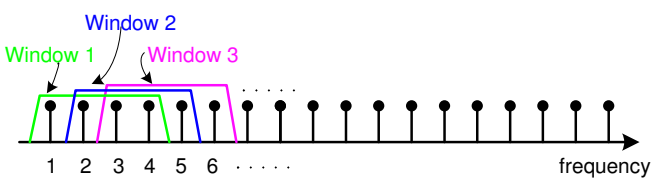

Fig. 2. Subcarrier power window.

For example, consecutive windows of subcarriers are shown in Fig. 2. If the power level of a subcarrier in 'Window 1' is lowered, the power can only be transferred to the other active subcarriers within 'Window 1'. When the power level of a subcarrier (say 'Subcarrier 3') is adjusted, subcarrier power level must satisfy the window power constraints for all the member sliding windows ('Window 1', 'Window 2', 'Window 3').

A practical transmit power constraint is usually enforced to limit the total power across a frequency window of a specified width. For instance, FCC has imposed requirements based on the amount of transmit power across a specified bandwidth in the UNII band [19]. These requirements are imposed since these bands are usually unlicensed and the users are non-cooperative.

\section{Proposed Algorithm}

To ensure the system transmissions do not violate regulatory requirements, we propose a subcarrier power adjustment PAPR reduction algorithm employing a window power constraint approach. The proposed subcarrier power adjustment algorithm for reducing PAPR is presented in Alg. 1. First, the algorithm begins with choosing the subcarrier power adjustment factors with a given distribution, $f(x)$. Second, either $L$ different sets of power adjustment factors are generated or the power adjustment factors are interleaved using $L$ interleavers. Third, each subcarrier power adjustment vectors are then window power-constrained using the algorithm presented in Alg. 2. Fourth, the input subcarriers are multiplied element-by-element with the subcarrier power adjustments. Finally, among the subcarrier power adjustment vectors, one yielding minimum PAPR is chosen.

The sliding window algorithm to maintain the power constraint over the window is presented in Alg. 2. First, the algorithm determines if the total power of the subcarriers in the $k$-th window, $\sum_{l=0}^{M-1} \pi(l)$, exceeds the practical power constraint, where $M$ represents the total number of subcarriers per window. If the total power of the subcarriers in a given window crosses allowable power level, the subcarrier power levels are scaled down to meet the power requirement. Second, the algorithm determines whether the power level of the $l$-th subcarrier, 

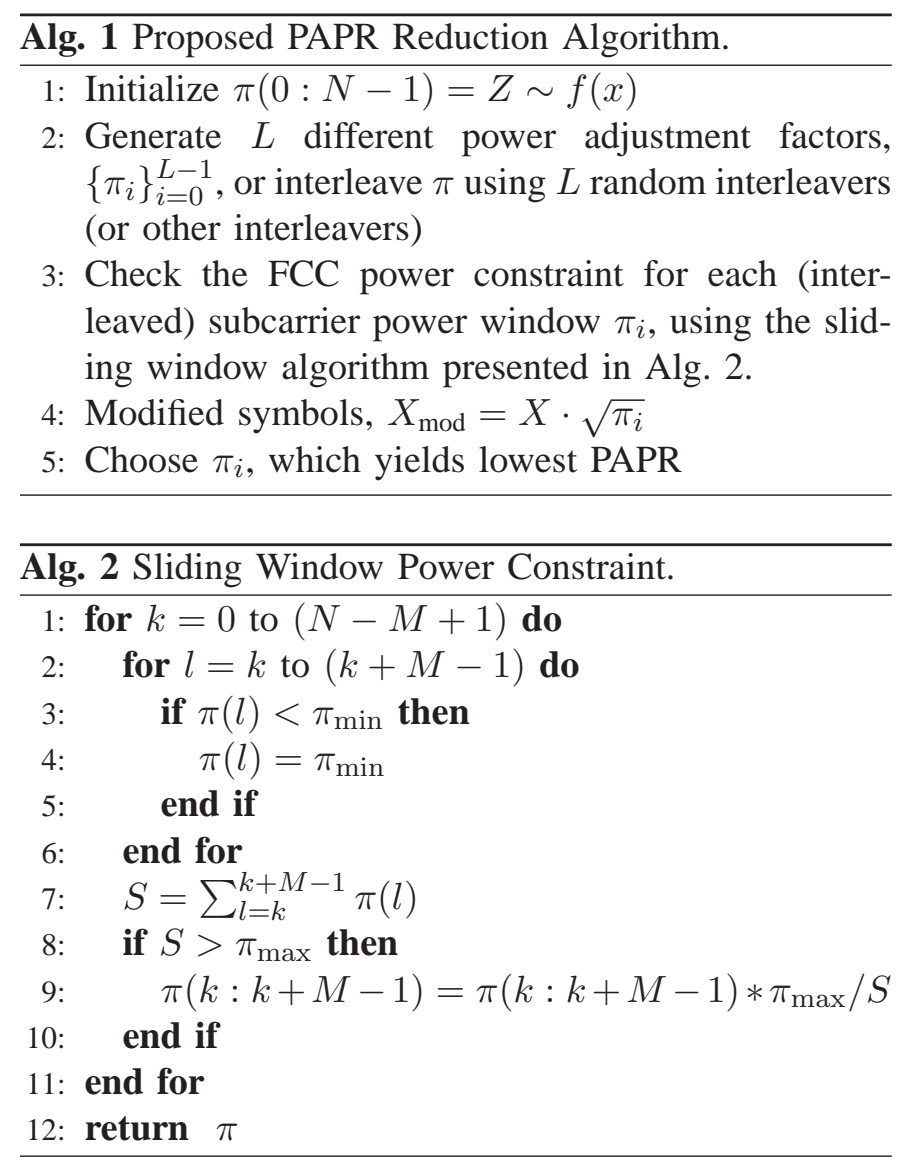

$\pi(l)$, is lower than the predefined threshold, $\pi_{\min }$, in which case, the power level is restored to $\pi_{\min }$. This minimum power level requirement would ensure that the BER performance would not degrade below certain level. The algorithm is repeated until both requirements are met by all the subcarriers.

The primary advantage of the proposed algorithm is that subcarrier power information need not to be transmitted to the receiver ${ }^{4}$. Therefore, there would not be any throughput loss and no modification would be necessary in receiver structure. Additionally, the subcarrier power levels can be quantized or set to the one digit decimal to reduce the complexity (or hardware requirements) of multiplication operations.

\section{Vi. Simulation Results}

\section{A. Simulation Setup}

For simulation purposes, OFDM transceiver employing 256 QPSK-modulated subcarriers is considered. To obtain each CCDF plots, $10^{4}$ random OFDM symbols are

\footnotetext{
${ }^{4}$ In MPSK modulated OFDM system, inverting the subcarrier power adjustment at the receiver would weight noise power as well, which keeps SNR unchanged.
}

generated and PAPR is computed for each symbol. The number of subcarriers per sliding window is assumed to be four. In this paper, we consider the following three subcarrier power level distributions:

1) Uniform: $f(x)=U(x), \quad x \geq 0$

2) Exponential: $f(x)=-\log (U(x)), \quad x \geq 0$

3) Truncated Gaussian: $f(x)=N\left(1, \sigma^{2}\right), \quad x \geq 0$

For all distributions, mean power is kept unity for fair comparison. We use $L=16$ random interleavers to generate different sets of power adjustment factors.

\section{B. PAPR Results}

For uniform, exponential, and truncated Gaussian subcarrier power distributions, CCDF of the PAPR obtained through simulations are presented in Fig. 3. The CCDF of PAPR is evaluated for each case with minimum subcarrier amplitude level, i.e. $\sqrt{\pi_{\min }}$, equal to $60 \%$, $70 \%, 80 \%$, and $90 \%$, and compared with original case. From Fig. 3, it is observed that PAPR gets reduced when the minimum subcarrier power level, $\pi_{\min }$, is reduced. This is because of the higher range of variantion among the subcarrier power levels. There is about $1.5 \mathrm{~dB}$ PAPR reduction at $10^{-3} \mathrm{CCDF}$ achieved when the subcarrier power distribution is exponential or truncated Gaussian, whereas there is about $0.5 \mathrm{~dB}$ PAPR reduction at $10^{-3}$ CCDF achieved when the subcarrier power distribution is uniform.

Assuming AWGN channel, BER performance degradation due to subcarrier power adjustments for uniform, exponential, and truncated Gaussian subcarrier power distributions are presented in Fig. 4. BER performance of the OFDM system is evaluated for each case with minimum subcarrier amplitude level, i.e. $\sqrt{\pi_{\min }}$, equal to $60 \%, 70 \%, 80 \%$, and $90 \%$, and compared with original case. The results corroborates the fact that the BER performance of the system degrades as the minimum subcarrier power level gets reduced. However, the BER performance degradation is relatively indifferent to the subcarrier power distributions.

From the results, it is ensured that there exists a tradeoff between the PAPR reduction and BER performance degradation. Higher PAPR reduction can be achieved when the minimum subcarrier amplitude level is reduced, but BER performance also degrades side-by-side. If slight degradation in BER performance is acceptable, significant PAPR reduction can be achieved by the proposed algorithm without increasing the complexity of the OFDM receivers. 


\section{CONCLUSION}

In this paper, we propose a novel subcarrier power adjustment algorithm for PAPR reduction that does satisfy spectrum regulation requirements. The proposed algorithm achieves large reduction in PAPR at the cost of slight degradation in aggregate BER performance of the system. The primary advantage of the proposed algorithm is that no overhead information needs to be transmitted to the receiver when the OFDM transceiver employs MPSK modulation. Therefore, no throughput loss and no additional processing are required at the receiver side.

\section{REFERENCES}

[1] J. A. C. Bingham, ADSL, VDSL, and Multicarrier Modulation. New York, USA: John Wiley and Sons Inc., 2000.

[2] Institute of Electronics and Electrical Engineers, "Wireless LAN medium access control(MAC) and physical layer (PHY) specifications: High speed physical layer in $5 \mathrm{GHz}$ band." IEEE Standard 802.11a, Nov. 1999.

[3] Institute of Electronics and Electrical Engineers, "Wireless LAN medium access control(MAC) and physical layer (PHY) specifications: Further higher-speed physical layer extension in $2.4 \mathrm{GHz}$ band." IEEE Standard 802.11g, June 2003.

[4] Institute of Electronics and Electrical Engineers, "Air interface for fixed broadband wireless access systems: Medium access control modifications and additional physical layer specifications for 2-11 GHz." IEEE Standard 802.16a, June 2004.

[5] L. J. Cimini Jr., "Analysis and simulation of a digital mobile channel using orthogonal frequency division multiplexing," IEEE Trans. Commun., vol. 33, pp. 665 - 675, July 1985.

[6] N. Y. Ermolova and P. Vainikainen, "On the relationship between peak factor of a multicarrier signal and aperiodic autocorrelation of the generating sequence," IEEE Commun. Lett., vol. 7, pp. 107-108, Mar. 2003.

[7] C. Tellambura, "Upper bound on peak factor of N-multiple carriers," Electron. Lett., vol. 33, pp. 1608-1609, Sept. 1997.

[8] J. Tellado, Multicarrier modulation with low PAR: Applications to DSL and Wireless. Massachusetts, USA: Kluwer Academic Publishers, 2000.

[9] P. Foomooljareon, W. A. C. Fernando, and K. M. Ahmed, "PAPR reduction of OFDM systems using input sequence envelope scaling," in Proc. 57th IEEE Veh. Technol. Conf. Spring, vol. 2, pp. 1243 - 1247, Apr. 2003.

[10] H. Nikookar and R. Prasad, "Weighted OFDM for wireless multipath channels," IEICE Trans. Commun., vol. E83-B, pp. 18641872, Aug. 2000.

[11] I. A. Tasadduq and R. K. Rao, "Weighted OFDM with block codes for wireless communication," in Proc. IEEE Pacific Rim Conf on Comm., Computers and Signal Processing, vol. 2, pp. 441-444, 2001.

[12] T. A. Thomas, "PAPR reduction via a fixed frequency-domain weighting across multiple OFDM bauds," in Proc. IEEE Int. Conf. Acoust., Speech, Signal Process., pp. 1081-1084, May 2004.

[13] B. S. Krongold and D. L. Jones, "PAR reduction in OFDM via active constellation extension," IEEE Trans. Broadcast., vol. 49, pp. $258-268$, Sept. 2003.
[14] C. Tellambura, "Computation of the continuous time PAR of an OFDM signal with BPSK subcarriers," IEEE Commun. Lett., vol. 5, pp. 185-187, May 2001.

[15] H. Yu and G. Wei, "Computation of the continuous time PAR of an OFDM signal," in Proc. IEEE Int. Conf. Acoust., Speech, Signal Process., vol. 2003, (Hong Kong), pp. IV - 529-31, Apr. 2003.

[16] A. M. Wyglinski, Physical layer loading algorithms for indoor wireless multicarrier systems. PhD thesis, McGill University, Montreal, Canada, Nov. 2004.

[17] I. Kalet, "The multione channel," IEEE Trans. Commun., vol. 37, pp. 119-124, Feb. 1989.

[18] R. F. H. Fischer and J. B. Huber, "A new loading algorithm for discrete multitone transmission," in Proc. IEEE Global Telecommun. Conf., vol. 1, (London, UK), pp. 724-728, Nov. 1996.

[19] Federal Communications Commission, "Part 15 - radio frequency devices, subpart E - unlicenced national information infrastructure devices, section 403 - definitions." Code of Federal Regulations - FCC 47CFR15.403, Oct. 2000.

[20] J. A. C. Bingham, "Multicarrier modulation for data transmission: an idea whose time has come," IEEE Commun. Mag., vol. 28, pp. 5-14, May 1990. 


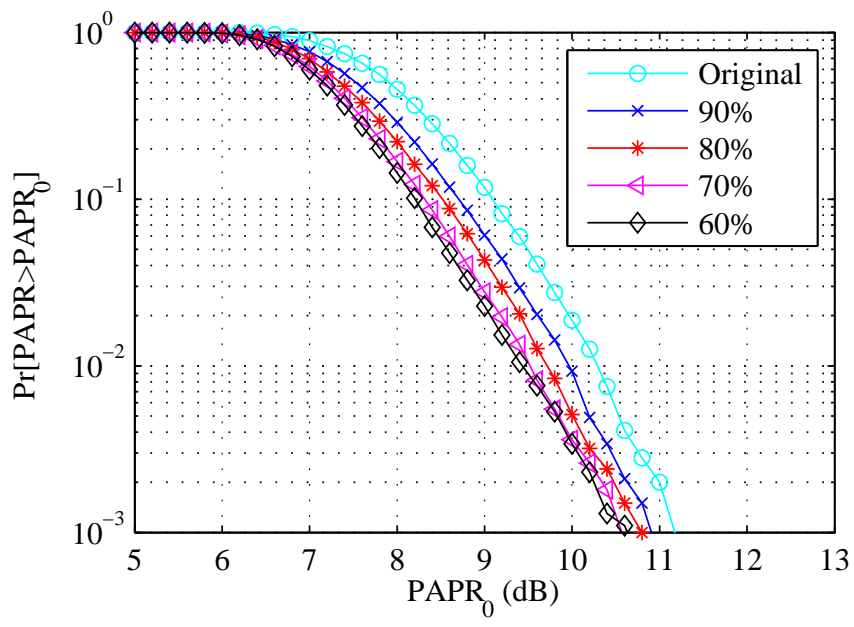

(a) Uniform Distribution.

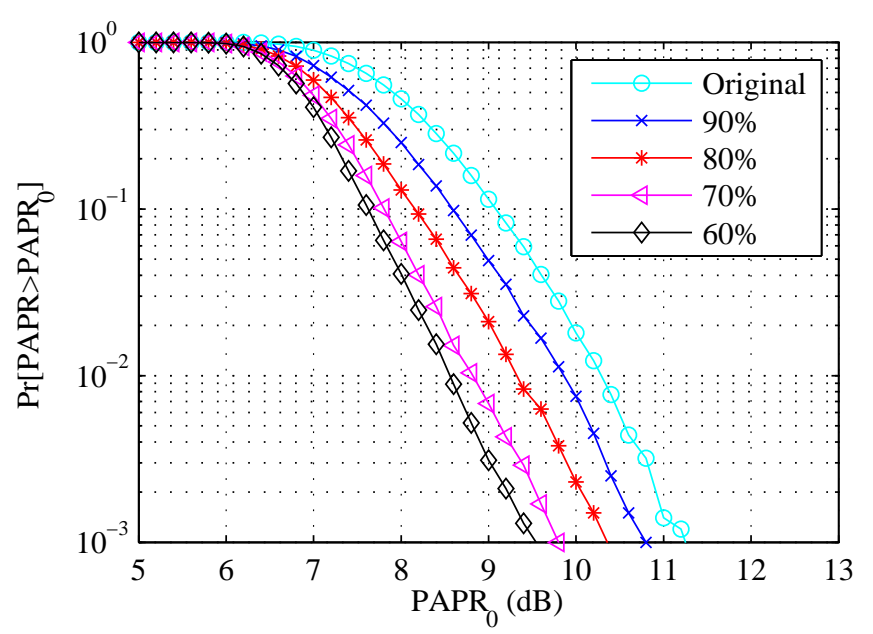

(b) Exponential Distribution.

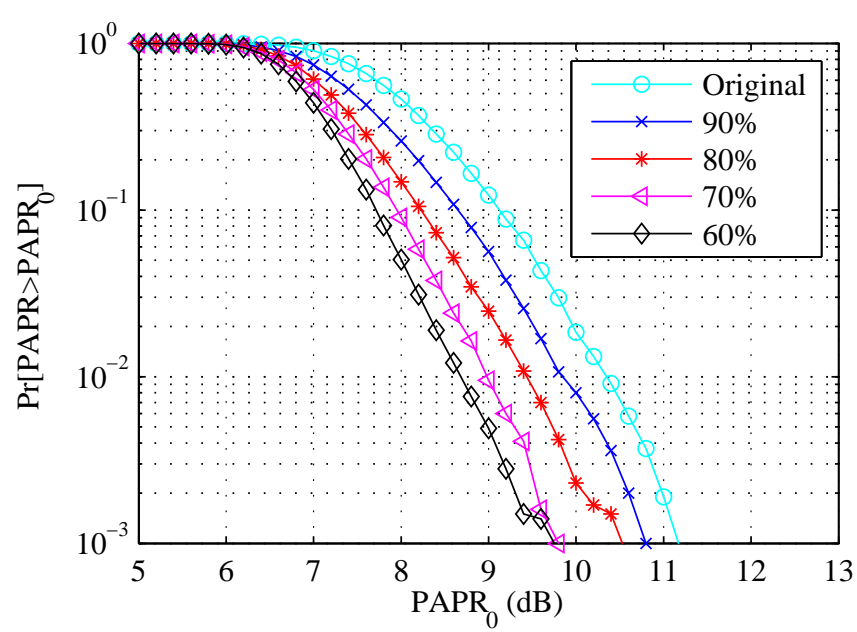

(c) Truncated Gaussian Distribution.

Fig. 3. Complementary cumulative distribution function of PAPR for 3 modulated OFDM with 256 subcarriers. QP SK

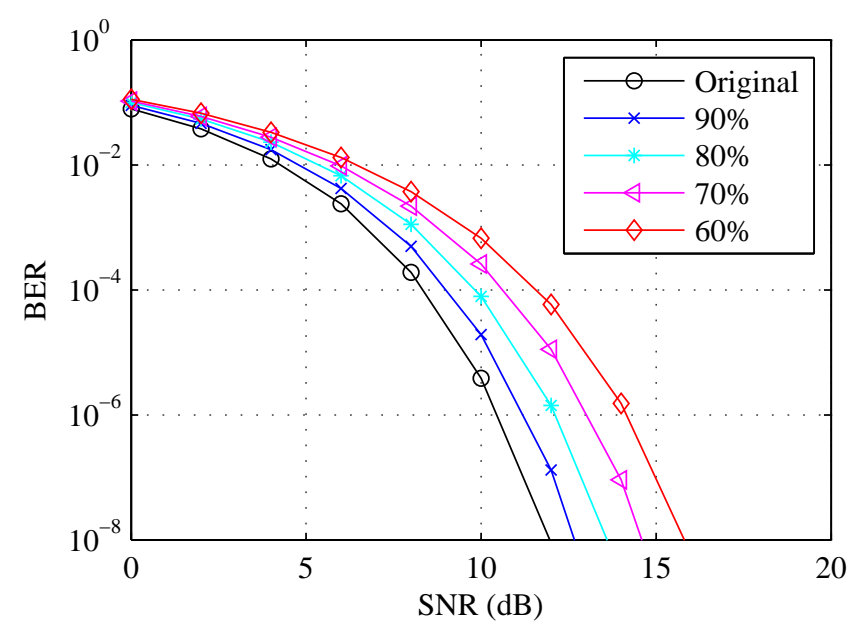

(a) Uniform Distribution.

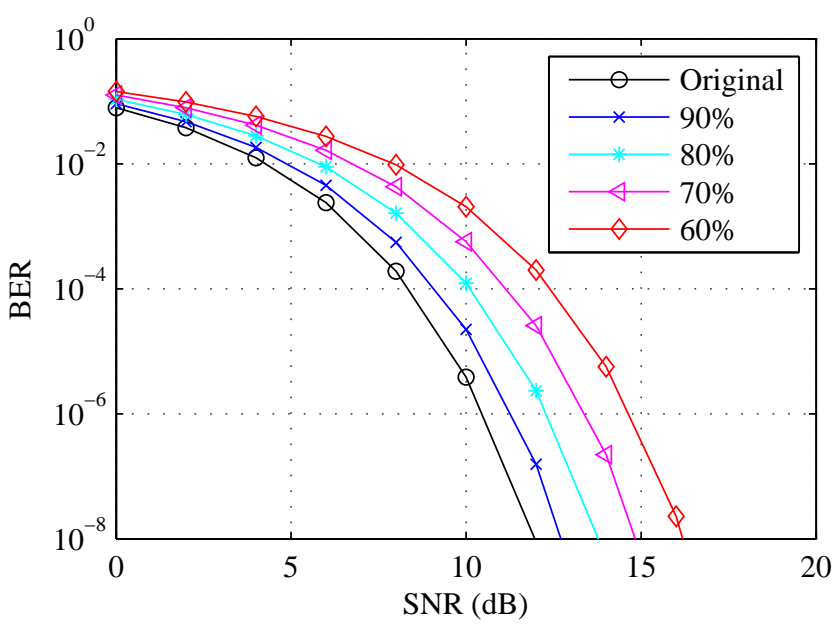

(b) Exponential Distribution.

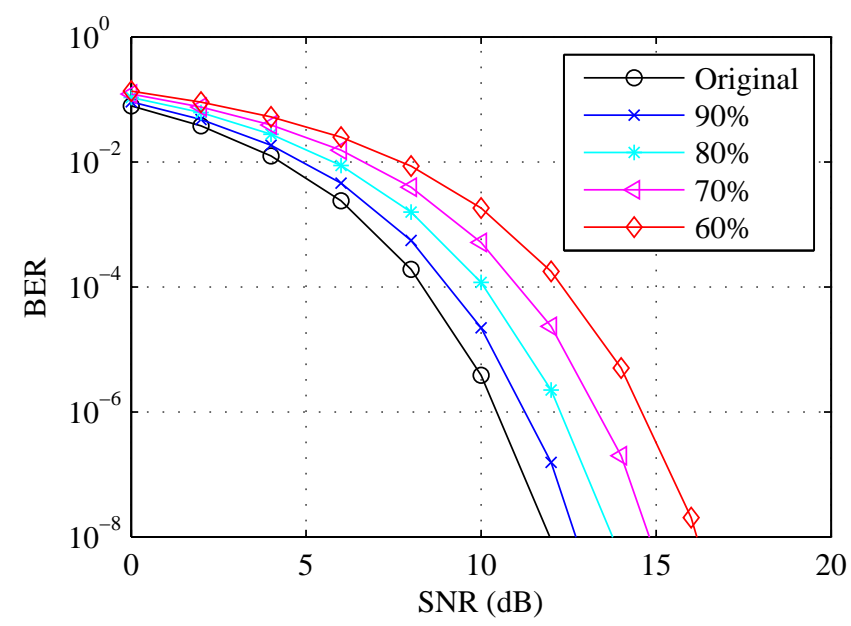

(c) Truncated Gaussian Distribution.

QPSK

Fig. 4. Aggregate BER performance of modulated OFDM with 256 subcarriers over AWGN channel. 